\title{
Extracting the Spin and Valley Information of Electrons
}

\author{
An electron's spin and valley information can be determined by \\ monitoring how easily that electron passes through a qubit.
}

\author{
By Rachel Berkowitz
}

$\prod$

he state of an electron-spin qubit depends primarily on the spin of the electron it houses. For some materials, the state may also depend on the electron's "valley degree of freedom," which relates to the local extremes of a material's electronic band structure. But obtaining this spin and valley-degree-of-freedom information is difficult. Now, using a system made of coupled graphene quantum dots, Chuyao Tong, of the Swiss Federal Institute of Technology (ETH) in Zurich, and her colleagues demonstrate how to extract an electron's spin and valley information from how easily that electron passes through the device [1]. Their approach underlines the potential for gaining control of the state of coupled graphene quantum dots, which would make them more useful for quantum technology applications.

A system consisting of two coupled quantum dots can be tuned so that it sits in a regime where it simultaneously lives in two states. In one of these states there is one electron in each dot. In the other, both electrons reside in one dot, and the other dot is

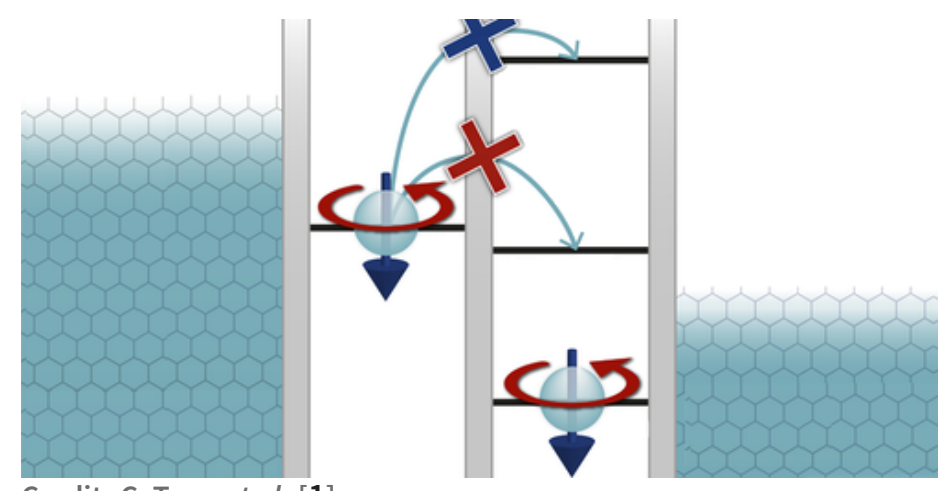

Credit: C. Tong et al. [1] empty. The transfer of an electron into a dot that already contains an electron is only successful if the two electrons have spin and valley numbers that match those of the system's ground state. If an electron has those numbers, it can pass in and out of the dot, causing a current to flow in the system. If not, the electron gets "blocked," and no current flows.

In their experiments, Tong and her team show that they can use a magnetic field to switch between two different single-dot, two-particle ground states, one selecting the valleys and one selecting the spins.

The researchers created a coupled-electron double quantum dot defined by electrostatic gates in bilayer graphene, to which they applied a magnetic field in the direction perpendicular to the graphene sheets. First they passed electrons through the coupled quantum dot. Then they measured the resulting current. Because the two-particle ground states were deducible, and the state transitions were related to the magnitude of the applied magnetic field, the team was able to infer whether the blockade was caused by the electron's spin or valley number.

Rachel Berkowitz is a Corresponding Editor for Physics based in Vancouver, Canada.

\section{REFERENCES}

1. C. Tong et al., "Pauli blockade of tunable two-electron spin and valley states in graphene quantum dots," Phys. Rev. Lett. 128, 067702 (2022). 